\title{
The evaluation of cellular immune function in elderly patients with systemic lupus erythematosus
}

\author{
Kun $\mathrm{Men}^{1}$, Yu Chen ${ }^{2}$, Jingwei Zhang ${ }^{1}$, and Dianjun $\mathrm{Wei}^{1}$
}

Departments of ${ }^{1}$ Clinical Laboratory and ${ }^{2}$ Endocrinology, The Second Hospital of Tianjin Medical University, Tianjin, China

Received: July 3, 2017

Revised : July 27, 2017

Accepted: August 7, 2017

\section{Correspondence to} Dianjun Wei, Ph.D.

Department of Internal Medicine, Department of Clinical Laboratory, The Second Hospital of Tianjin Medical University, No. 23 PingJiang Rd, He'xi Dist, Tianjin, 300211, China

Tel: $+86-22-88328930$

Fax: +86-22-88326837

E-mail: dianjun_wei@163.com
Background/Aims: To evaluate cellular immune function in systemic lupus erythematosus (SLE) patients over 60 years old, the association between antinuclear antibody (ANA) titers and the ratio of $\mathrm{CD}_{4}^{+} / \mathrm{CD}^{+}$was analyzed in this study. The distribution of ANAs and extractable nuclear antibodies (ENAs) in a healthy elderly population was also investigated.

Methods: Serum ANA titers were assayed by indirect immunofluorescence (IIF) and the $\mathrm{CD}_{4}^{+} / \mathrm{CD}^{+} \mathrm{T}$-cell ratio was determined by flow cytometry in 76 SLE patients and 30 healthy control individuals. IIF of cytoplasm and nuclear and nucleolar staining were performed on samples taken from 286 healthy elderly individuals. ENA levels were determined using a strip enzyme immunoassay among patients that tested positive for ANAs.

Results: ANA titers were negative in the 30 control individuals, but were positive in the 76 SLE patients. Based on ANA titers, the SLE patients were stratified to low $(\leq 1: 320)$, medium (1:640 to 1:1,280), and high $(\geq 1: 2,560)$ titer groups. The average $\mathrm{CD}_{4}+/ \mathrm{CD}^{+}$ratio of the SLE group was significantly lower than that of the control group. Among the 286 healthy elderly volunteers, 59 (20.63\%) tested positive for ANAs. A homogeneous pattern was present in $47.46 \%$ of those 59 patients and a granule pattern in the karyoplasm was present in $33.90 \%$. Furthermore, of the 59 patients, ENAs immunoassay was positive in 18 (30.51\%); Sjogren syndrome-related antigen A (SSA)/52 kd and Sjogren syndrome antigen B (SSB)/La were the two major antibodies.

Conclusions: The significantly lower $\mathrm{CD}_{4}^{+} / \mathrm{CD}^{+}$ratio among SLE patients over 60 years old is associated with deregulated immune responses and the development of SLE. A low ANA titer (1:160) is common in healthy elders, emphasizing the importance of considering age when determining if the evaluation of ANA titers is to be included in autoimmune disease diagnosis.

Keywords: Antibodies, antinuclear; Extractable nuclear antibody; Lupus erythematosus, systemic; CD4+ T-cells; CD8+ T-cells

\section{INTRODUCTION}

Systemic lupus erythematosus (SLE) is an autoimmune disease involving multiple systems [1]. Though the etiology of SLE is not completely understood, the involve- ment of T-cells in this kind of disease is being investigated [2]. Mature $\mathrm{CD}_{4}+$ and CD8+ T-cells, which can be distinguished by their surface $\mathrm{CD}$ antigens, maintain the balance of cellular and humoral immunity [3]. An imbalance of cellular and humoral immunity is close- 
ly associated with the development of SLE. Peripheral blood antinuclear antibodies (ANAs) target self-proteins in the nucleus of eukaryotic cells. The determination of ANA titers is widely appreciated in SLE diagnosis [4]. The indirect immunofluorescence (IIF) technique on monolayers of cultured epithelial cells is the recommended screening method for the detection of ANAs because it has higher sensitivity than solid phase assays, and it plays an important role in immunological assays for detecting and measuring autoantibodies [5]. The presence of ANAs in peripheral blood is positively correlated with SLE when the titer is greater than 1:160, so the $\mathrm{CD}_{4}{ }^{+} /$ $\mathrm{CD} 8^{+}$ratio might thus assist in definitive SLE diagnoses [6]. In this study, the $\mathrm{CD}_{4}^{+} / \mathrm{CD}^{+}$ratio and its relationship with ANA titers is determined to evaluate the status of immune cell functions in SLE patients. Furthermore, SLE epidemics in healthy elders in Tianjin city is monitored based on ANA titers.

\section{METHODS}

\section{Patients}

From September 2013 to November 2013, 76 SLE inpatients over 60 years old were admitted to the Second Hospital of Tianjin Medical University. SLE was diagnosed following the 2011 American College of Rheumatology criteria. Patients were stratified by their ANA titers into low ( $\leq 1: 320)$, medium (1:640 to 1:1,280), or high ( $\geq 1: 2,560)$ groups. The characteristics of the 76 SLE patients and 30 healthy control participants are shown in Table 1. A total of 30 healthy control participants and another group of 286 healthy elderly individuals, including 237 men aged 70 to 96 years old and 49 women aged 71 to 95 years old who met China elderly health standards, were recruited at the Second Hospital of Tianjin Medical University in October 2013. None of these individuals had been diagnosed with serious diseases or conditions such as cancer, aortic valve stenosis, diabetes, or transient ischemic attack. There was no statistical significance in gender and age $(p>0.05)$ between the group and the data was comparable. Age and sex in the control group were matched. Informed consent was obtained from all individual study participants and their families. This study was also approved by the Research Ethics Committee (201306-011) of The Second Hospital of Tianjin Medical University (Tianjin, China).

\section{$\mathrm{CD}_{4}{ }^{+} / \mathrm{CD} 8^{+}$lymphocyte ratio}

Flow cytometry was used to assay $\mathrm{CD}_{4}{ }^{+}$and $\mathrm{CD} 8^{+}$lymphocyte populations. The fluorescein isothiocyanate (FITC)-CD $4^{+}$and phycoerythrin (PE)-CD8 ${ }^{+}$conjugated monoclonal antibodies were purchased from Abcam (Cambridge, UK). The erythrocyte lysis buffer was purchased from Sigma-Aldrich (Munich, Germany). Venous blood samples $(1 \mathrm{~mL})$ were collected from each participant and transferred to three tubes (control, FITC-CD $4{ }^{+}$, and PE-CD $\left.8^{+}\right)$. For flow cytometry assay, blood $(100 \mu \mathrm{L})$ and monoclonal antibody $(20 \mu \mathrm{L})$ were added to each tube and mixed by gentle shaking. The tubes were kept at room temperature for addition of the erythrocyte lysis buffer. The lysate was centrifuged at 1,500 $\mathrm{xg}$ for $5 \mathrm{~min}$ utes and the supernatants were removed. The pellet was resuspended in phosphate-buffered saline (PBS). The lysate was centrifuged again at 1,500 $\mathrm{xg}$ for 5 minutes, the supernatants were removed, and the pellet was resuspended in $500 \mathrm{~mL}$ of PBS for assay (Coulter XL FCM, Beckman Coulter, Brea, CA, USA).

\section{ANA titers}

The ANA titers were assayed by IIF technique with a commercially available kit (ANAs pattern kit, Beijing HOB Biotech, Beijing, China). After a morning collection of venous blood, samples were centrifuged at 3,000 $x g$ for 5 minutes to obtain serum. The ANA titers of 286 serum samples were determined using the ANAs pat-

Table 1. Participants characteristics

\begin{tabular}{lccc}
\hline Group & Number & Men/Women & Age, yr, mean \pm SD \\
\hline Control & 30 & $18 / 12$ & $62 \pm 2$ \\
Low titer & 31 & $12 / 19$ & $64 \pm 3$ \\
Medium titer & 25 & $11 / 14$ & $62 \pm 2$ \\
High titer & 20 & $10 / 10$ & $64 \pm 3$ \\
\hline
\end{tabular}


Table 2. The $\mathrm{CD}_{4}^{+} / \mathrm{CD}^{+}$ratio in each group

\begin{tabular}{lccr}
\hline Group & Number & Male/Female & $\mathrm{CD}^{+} / \mathrm{CD}^{+} \mathrm{ratio}^{\mathrm{mean} \pm \mathrm{SD}}$ \\
\hline Control & 30 & $18 / 12$ & $1.40 \pm 0.66$ \\
Low titer & 31 & $12 / 19$ & $1.13 \pm 0.47$ \\
Medium titer & 25 & $11 / 14$ & $0.99 \pm 0.23$ \\
High titer & 20 & $10 / 10$ & $0.54 \pm 0.18$ \\
\hline
\end{tabular}

Table 3. Between-group comparisons of $\mathrm{CD}_{4}^{+} / \mathrm{CD}^{+}$ratio

\begin{tabular}{|c|c|c|c|c|}
\hline Group & Control & Low titer & Medium titer & High titer \\
\hline Control & NA & $0.022^{\mathrm{a}}$ & $0.001^{\mathrm{a}}$ & $0.000^{\mathrm{a}}$ \\
\hline Low titer & $0.022^{\mathrm{a}}$ & NA & 0.233 & $0.000^{\mathrm{a}}$ \\
\hline Medium titer & $0.001^{\mathrm{a}}$ & 0.233 & NA & $0.001^{\mathrm{a}}$ \\
\hline High titer & $0.000^{\mathrm{a}}$ & $0.000^{\mathrm{a}}$ & $0.001^{a}$ & NA \\
\hline
\end{tabular}

NA, not applicable.

${ }^{a} p<0.05$.

tern kit following the manufacturer's protocol. The ANA titers of each sample were determined by comparison to the standards included in the ANAs pattern kit, and then were read using a BX41 fluorescence microscope (Olympus, New York, NY, USA). When the ANA titers were more than 1:160, the sample was considered positive. The extractable nuclear antibodies (ENAs) of ANA-positive samples were determined by comparison to standards included in a commercially available ENAs kit using a strip enzyme immunoassay following the manufacturer's protocol (Beijing HOB Biotech).

\section{Statistical analysis}

SPSS version 17.0 software (SPSS Inc., Chicago, IL, USA) was used for general statistical analysis. The normality of the distribution of $\mathrm{CD}_{4}{ }^{+/} \mathrm{CD} 8^{+}$ratio was examined using a histogram. Pairwise comparisons were tested for significance by analysis of variance and least significance distance (LSD) statistics. Data were expressed as the mean \pm standard deviation $(\mathrm{SD})$. The results were considered significant when the $p$ value was less than 0.05 .

\section{RESULTS}

\section{ANA titers and the $\mathrm{CD}_{4}{ }^{+} / \mathrm{CD} 8^{+}$ratio}

ANA titers were not detectable (negative) among all 30

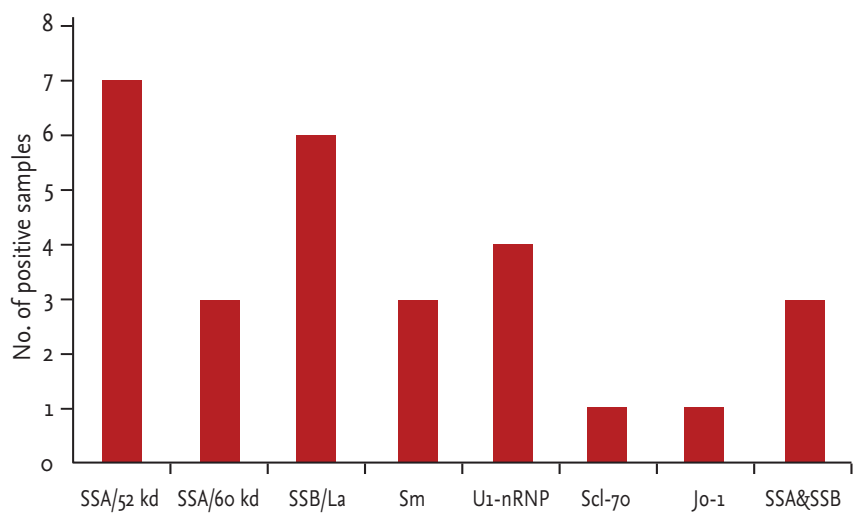

Figure 1. Extractable nuclear antibodies (ENAs) were determined using a strip enzyme immunoassay among antinuclear antibody-positive patients. The most frequent ENAs were Sjogren syndrome-related antigen A (SSA)/52 kd and Sjogren syndrome antigen B (SSB)/La, followed by Sm, U1nRNP, Scl-7o, and Jo-1, respectively.

control subjects. All 76 SLE patients had measurable ANA titers and were stratified by their titers into low ( $\leq 1: 320)$, medium (1:640 to 1:1,280), and high ( $\geq 1: 2,560)$ groups. The $\mathrm{CD}_{4}^{+} / \mathrm{CD} 8^{+}$ratio decreased as the ANA titers increased (Table 2). The values of $\mathrm{CD}_{4}^{+} / \mathrm{CD} 8^{+}$ratio were normally distributed. The F-value of the variance between groups was 14.989. LSD analysis indicated that the reduction of the $\mathrm{CD}_{4}+\mathrm{CD} 8^{+}$ratio observed with increased ANA titers was significant (Table 3). 


\section{ANA titers in healthy elderly individuals}

ANA titers were measureable (positive) in 59 of the 286 healthy elderly people $(20.63 \%)$, with titers ranging from 1:160 to 1:1,280. Among the ANA-positive participants, $68 \%$ had low titers (1:160). Those participants with a homogeneous pattern (47.46\%) and granular pattern $(33.90 \%)$ in the cytoplasm accounted for the majority, whereas nucleolar, anti-centromere, and cytoplasmic granule patterns were seen less frequently. ENAs were detected in 18 of the 59 ANA-positive participants (30.5\%), and many of these individuals were positive for two or more antibodies. The most frequently seen ENAs were SSA/52 kd and SSB/La, followed by Sm, U1-nRNP, Scl-70, and Jo-1, respectively (Fig. 1).

\section{DISCUSSION}

The immune system plays an important role during the progression of SLE. T-cells mediate both cell and hue moral immunity, and an imbalance between $\mathrm{CD}_{4}{ }^{+}$and $\mathrm{CD} 8^{+} \mathrm{T}$-cell function disturbs immune homeostasis and immune system response. $\mathrm{CD}_{4}^{+} \mathrm{T}$-cells, also referred to as Thelper (Th) cells, play a key role in adaptive immune response, attracting and activating other immune cells [7]. Recent evidence links SLE and other autoimmune diseases with abnormal expression of $\mathrm{CD}_{4}{ }^{+} \mathrm{T}$-cells $[8,9]$. $\mathrm{CD}_{4}{ }^{+} \mathrm{T}$-cells can prompt B lymphocytes to produce immunoglobulin $\mathrm{G}$ antibodies under pathologic conditions, and the antibodies can bind to self-antigens in multiple organs and tissues. Binding may occur at sites like the glomerular basement membrane, liver, central nervous system, and small vessel walls in the form of immune complexes that produce vascular inflammation and blockage [10]. CD8 ${ }^{+} \mathrm{T}$-cells, also referred to as killer T-cells, produce toxic granules that induce apoptosis and death of pathogen-carrying cells [11]. A recent study has shown that a change in number and function of inhibitory T-cells could affect the development of autoimmune diseases [12]. Helper T lymphocytes activate autoreactive $\mathrm{B}$ lymphocytes. Excessive activation of autoreactive B lymphocytes is thus T-cell-dependent, which suggests the disorder of $\mathrm{T}$-cells functions in the development of SLE.

In this study, the $\mathrm{CD}_{4}{ }^{+} / \mathrm{CD} 8^{+}$cell ratio is significantly lower in SLE patients than it is in the healthy control participants. This low ratio indicates a poor immune response. The absolute numbers of $\mathrm{CD}_{4}{ }^{+}$or $\mathrm{CD} 8^{+}$cells influence SLE disease progression, and functions of these cells also plays a part in the similar regulation. This idea has been proven by various studies $[13,14]$. However, the use of $\mathrm{CD}_{4}{ }^{+} / \mathrm{CD}^{+}$cell ratio as a maker to evaluate cell-mediated immunity remains relatively rare. Maeda et al. [15] demonstrated that the $\mathrm{CD}_{4}{ }^{+} / \mathrm{CD}^{+}$ ratio was decreased in SLE patients and that this was significantly related to expression of human leukocyte antigen (HLA)-DR by CD $8^{+}$(but not $\mathrm{CD}_{4}^{+}$) T-cells, which indicates that the $\mathrm{CD}_{4} / \mathrm{CD}^{+}$ratio may be a good indicator of therapeutic efficacy in some SLE patients. In the current research, the $\mathrm{CD}_{4}^{+} / \mathrm{CD}^{+}$ratio was significantly lower in the SLE group than in the control group, and it is associated with the development of SLE based on the speculation that the change in the $\mathrm{CD}_{4}^{+} / \mathrm{CD}^{+}$ ratio disrupts cellular immunity thus leading to SLE. Mounting reports have shown that either $\mathrm{CD}_{4}{ }^{+}$or $\mathrm{CD} 8+$ is closely correlated with systemic lupus erythematosus disease activity index (SLEDAI) score and clinical manifestations. Zhao et al. [16] reported that the percentages of $\mathrm{CD}_{3}{ }^{+} / \mathrm{CD}_{4}{ }^{+} \mathrm{T}$-cells and the $\mathrm{CD}_{4}{ }^{+} / \mathrm{CD}^{+}{ }^{+} \mathrm{T}$-cell ratio were significantly decreased in SLE patients versus the control participants. The lymphocyte subsets were significantly correlated with the SLEDAI scores and complement factors $\left(\mathrm{C}_{3}, \mathrm{C}_{4}\right)$. The significant correlation between lymphocytes subsets and SLEDAI scores suggests that the lymphocyte subsets may reflect SLE disease severity [16]. Matsushita et al. [17] found that a change was observed in $\mathrm{CD}_{4}^{+} / \mathrm{CD}^{+}$ratio on T-cells with the improvement of clinical manifestations upon treatment with steroids or cyclosporine. In this study, the $\mathrm{CD}_{4}^{+}$/ $\mathrm{CD} 8^{+}$ratios in the low and medium titer groups were higher than the $\mathrm{CD}_{4}^{+} / \mathrm{CD}^{+}$ratio of the high titer group. There was little difference between the low and medium titer groups. This indicates that ANA titers are associated with the $\mathrm{CD}_{4}^{+} / \mathrm{CD}^{+}$ratio. Both the $\mathrm{CD}_{4}^{+} / \mathrm{CD}^{+}$ ratio and immune responses decreased with an increase of ANA titers. In summary, a change in the $\mathrm{CD}_{4}^{+} / \mathrm{CD}^{+}$ ratio could disturb cellular immunity and cause SLE. To a certain extent, ANA titers reflect cellular immune function in SLE, as the $\mathrm{CD}_{4}{ }^{+} / \mathrm{CD}^{+}$ratio and cellular immunity decreased as ANA titers increased.

Although ANA titers provide little clinical meaning for disease activity or prognosis, there are still many 
scholars who hold different views. For example, Wells et al. [18] showed that biopsy ANA appears to be a real phenomenon of unknown etiology, and not an artifact, which is found in some patients with active multisystem autoimmune disease, especially SLE. Song et al. [19] found that negative immunofluorescence anti-nuclear antibodies (IFANA) is mainly attributed to nephritic-range proteinuria, and large-dose glucocorticoid, immunosuppressant, and severe infection are also important factors for negative IFANA. ANA can be detected in some SLE patients with negative IFANA by changing the detection method and titer. Negative conversion of IFANA indicates unfavorable prognosis for severe patients [19]. Moreover, to study the clinical and laboratorial characteristics of patients with SLE, other markers such as double stranded DNA or SLEDAI will be included in a future study. A large number of samples are necessary to demonstrate the relationship between ANA titers and the $\mathrm{CD}_{4}^{+} / \mathrm{CD} 8^{+}$ratio.

The adaptive immune system encounters numerous antigens and is reconstituted repeatedly in the course of life [2o]. It is more active in youth than in adults, and weakens with age. Regression of adaptive immune response increases with age and is attributed to immune-deficiency, but seems to be associated with an imbalance resulting in the increase in the production of autoantibodies [21]. In this study, ANAs were positive in $20.63 \%$ (59 of 286 ) healthy elderly people. In a large epidemiological study, ANAs were positive in 13.8\% Americans and increased with age, reaching $19.2 \%$ in participants $>70$ years of age, which was similar to this study's findings [22]. Other studies have also found an increased ANA prevalence in the elderly population $[23,24]$. Approximately $68 \%$ of the 59 ANA-positive healthy elderly participants in our study had low ANA titers (1:160), which was consistent with a report by Nilsson et al. [23] that low ANA titers predominate in men $>85$ years of age. In this study, the ANA titers of SLE patients were all more than 1:320 and ANA titers of 1:160 was the maximum for the 289 healthy individuals. Research conducted by Leuchten et al. [4] showed that IIF-ANA has a high sensitivity for SLE diagnosis. ANA titers of 1:80 are sufficient for SLE classification criteria; that is, it is appropriate to formally test other classification criteria for SLE only if ANA titers of at least 1:80 have been found [4]. In older age groups, frequent apoptosis degrades the protein on cell surfaces and makes the cells targeted by ANAs, which causes further increase in ANAs. Additional studies in healthy elderly people could verify changes in the prevalence of ANAs that occur with aging, but large study samples are necessary. The 289 healthy people in this study were chosen from the retired senior official, and the number of male participants was more than the number of female participants. Since the representativeness of the data appears low, the number of the male and female participants will be counterweighed in a future study.

IIF in ANA-positive participants demonstrated granular, nucleolar, and cytoplasmic staining patterns. Among the 18 ENA-positive participants, the most frequently observed antigens were SSA/52 kd and SSB/La, which are associated with several autoimmune diseases. Positive staining of Sm, U1-nRNP, Scl-70, and Jo-1, which are associated with specific autoimmune diseases, were less frequently observed. An objective evaluation of SLE is based on a combination of the clinical symptoms and other indications, including current medications and presence of non-autoimmune diseases, while also considering age when ANA titers are used for diagnostic purposes.

In summary, the significantly lower $\mathrm{CD}_{4}^{+} / \mathrm{CD} 8^{+}$ratio among elderly patients is associated with deregulated immune responses and the development of SLE. Low ANA titers are common in healthy elders and should be considered during SLE diagnoses.

\section{KEY MESSAGE}

1. $\mathrm{CD}_{4}{ }^{+/} \mathrm{CD}^{+}$ratio was significantly decreased among systemic lupus erythematosus (SLE) patients over 60 years old.

2. The decreased $\mathrm{CD}_{4}^{+} / \mathrm{CD}^{+}$ratio was associated with deregulated immune responses and the development of SLE.

\section{Conflict of interest}

No potential conflict of interest relevant to this article was reported. 


\section{REFERENCES}

1. Wu H, Zhao M, Chang C, Lu Q. The real culprit in systemic lupus erythematosus: abnormal epigenetic regulation. Int J Mol Sci 2015;16:11013-11033.

2. Grammatikos AP, Ghosh D, Devlin A, Kyttaris VC, Tsokos GC. Spleen tyrosine kinase (Syk) regulates systemic lupus erythematosus (SLE) T cell signaling. PLoS One 2013;8:e74550.

3. Wu Y, Cai B, Feng W, et al. Double positive CD4+CD8+ T cells: key suppressive role in the production of autoantibodies in systemic lupus erythematosus. Indian J Med Res 2014;140:513-519.

4. Leuchten N, Hoyer A, Brinks R, et al. Performance of antinuclear antibodies for classifying systemic lupus erythematosus: a systematic literature review and meta-regression of diagnostic data. Arthritis Care Res (Hoboken) 2018;70:428-438.

5. Voigt J, Krause C, Rohwader E, et al. Automated indirect immunofluorescence evaluation of antinuclear autoantibodies on HEp-2 cells. Clin Dev Immunol 2012;2012:651058.

6. Choi BY, Yoon MJ, Shin K, Lee YJ, Song YW. Characteristics of pleural effusions in systemic lupus erythematosus: differential diagnosis of lupus pleuritis. Lupus 2015;24:321-326.

7. Zhu J, Paul WE. CD4 T cells: fates, functions, and faults. Blood 2008;112:1557-1569.

8. Zhang X, Liu F, Liu H, et al. Urinary trypsin inhibitor attenuates lipopolysaccharide-induced acute lung injury by blocking the activation of $\mathrm{p}_{3} 8$ mitogen-activated protein kinase. Inflamm Res 2011;60:569-575.

9. Suarez A, Lopez P, Gomez J, Gutierrez C. Enrichment of $\mathrm{CD}_{4}+\mathrm{CD}_{25}$ high $\mathrm{T}$ cell population in patients with systemic lupus erythematosus treated with glucocorticoids. Ann Rheum Dis 2006;65:1512-1517.

10. Shiozawa S. Pathogenesis of SLE and aiCD4T cell: new insight on autoimmunity. Joint Bone Spine 2012;79:428430.

11. Kim JS, Cho BA, Sim JH, et al. IL-7Ralow memory CD8+ $\mathrm{T}$ cells are significantly elevated in patients with systemic lupus erythematosus. Rheumatology (Oxford) 2012;51:1587-1594.

12. Xu A, Shi G, Liu F, Ge B. Caenorhabditis elegans mom-4 is required for the activation of the p38 MAPK signaling pathway in the response to Pseudomonas aeruginosa infection. Protein Cell 2013;4:53-61.

13. Sonawale A, Bohara V, Bichile LS. Evaluation of the association between $\mathrm{CD}_{4}, \mathrm{CD} 8$ and $\mathrm{CD} 25$ cell counts and SLE in active disease and in remission. J Assoc Physicians India 2017;65:37-42.

14. Goropevsek A, Gorenjak M, Gradisnik S, et al. STAT5 phosphorylation in $\mathrm{CD}_{4} \mathrm{~T}$ cells from patients with SLE is related to changes in their subsets and follow-up disease severity. J Leukoc Biol 2017;101:1405-1418.

15. Maeda N, Sekigawa I, Iida N, Matsumoto M, Hashimoto $\mathrm{H}$, Hirose $\mathrm{S}$. Relationship between $\mathrm{CD}_{4}^{+} / \mathrm{CD}^{+} \mathrm{T}$ cell ratio and $\mathrm{T}$ cell activation in systemic lupus erythematosus. Scand J Rheumatol 1999;28:166-170.

16. Zhao L, Jiang Z, Jiang Y, Ma N, Wang K, Zhang Y. Changes in immune cell frequencies after cyclophosphamide or mycophenolate mofetil treatments in patients with systemic lupus erythematosus. Clin Rheumatol 2012;31:951959 .

17. Matsushita M, Hayashi T, Ando S, et al. Changes of CD4/ CD8 ratio and interleukin-16 in systemic lupus erythematosus. Clin Rheumatol 2000;19:270-274.

18. Wells JV, Webb J, Van Deventer M, et al. In vivo anti-nuclear antibodies in epithelial biopsies in SLE and other connective tissue diseases. Clin Exp Immunol 1979;38:424435 .

19. Song LJ, Ding F, Liu HX, et al. Analysis of 15 patients with systemic lupus erythematosus manifesting with negative immunofluorescence anti-nuclear antibodies after treatment. Lupus 2012;21:919-924.

20. Pawelec G. Immunosenescence: impact in the young as well as the old? Mech Ageing Dev 1999;108:1-7.

21. Boren E, Gershwin ME. Inflamm-aging: autoimmunity, and the immune-risk phenotype. Autoimmun Rev 2004;3:401-406.

22. Satoh M, Chan EK, Ho LA, et al. Prevalence and sociodemographic correlates of antinuclear antibodies in the United States. Arthritis Rheum 2012;64:2319-2327.

23. Nilsson BO, Skogh T, Ernerudh J, et al. Antinuclear antibodies in the oldest-old women and men. J Autoimmun 2006;27:281-288.

24. Hurme M, Korkki S, Lehtimaki T, et al. Autoimmunity and longevity: presence of antinuclear antibodies is not associated with the rate of inflammation or mortality in nonagenarians. Mech Ageing Dev 2007;128:407-408. 